\title{
Magnetic Reversal and Thermal Stability of CoFeB Perpendicular Magnetic Tunnel Junction Arrays Patterned by Block Copolymer Lithography
}

Kun-Hua Tu${ }^{1}$, Eduardo Fernandez Martin ${ }^{1,2}$, Hamid Almasi ${ }^{3}$, Weigang Wang ${ }^{3}$, David Navas Otero ${ }^{4}$, George Liontos ${ }^{5}$, Dimitrios Moschovas ${ }^{5}$, Apostolos Avgeropoulos ${ }^{5}$, Caroline A. Ross ${ }^{1 *}$

\author{
${ }^{1}$ Department of Materials Science and Engineering, Massachusetts Institute of Technology, Cambridge \\ MA 02139, USA \\ ${ }^{2}$ Basque Center for Materials, Applications and Nanostructures (BCMaterials), Parque Tecnológico de \\ Bizkaia, Building 500, Derio, Spain \\ ${ }^{3}$ Department of Physics, University of Arizona, Tucson, AZ 85721, USA \\ ${ }^{4}$ IFIMUP-IN and Departamento de Física e Astronomia, Universidade do Porto, 4169-007 Porto, Portugal. \\ ${ }^{5}$ Department of Materials Science Engineering, University of Ioannina, University Campus-Dourouti, \\ 45110 loannina, Greece \\ *Email: caross@mit.edu
}

\begin{abstract}
Dense arrays of pillars, with diameters of 64 and $25 \mathrm{~nm}$, were made from a perpendicular CoFeB magnetic tunnel junction thin film stack using block copolymer lithography. While the soft layer and hard layer in the $64 \mathrm{~nm}$ pillars reverse at different fields, the reversal of the two layers in the $25 \mathrm{~nm}$ pillars could not be distinguished, attributed to the strong interlayer magnetostatic coupling. First order reversal curves were used to identify the steps that occur during switching, and the thermal stability and effective switching volume were determined from scan rate dependent hysteresis measurements.
\end{abstract}

\section{Introduction}

Magnetic tunnel junctions with perpendicular magnetic anisotropy (p-MTJ) are of interest in memory and computing applications ${ }^{1-3}$ because of their non-volatility and low critical current 
density for spin-transfer torque switching. Perpendicular MTJs typically consist of ultra-thin CoFeB layers sandwiching a MgO tunnel barrier. Perpendicular magnetic anisotropy (PMA) is promoted by growth of the CoFeB on a heavy metal layer such as Pt or Ta, resulting in strong interfacial anisotropy, and the p-MTJs exhibit high tunneling magnetoresistance (TMR) (>120\%), low switching current, and high thermal stability. The growth ${ }^{4-7}$, the physical origin of interfacial anisotropy ${ }^{8}$, and voltage-controlled magnetic properties ${ }^{9-11}$ of $\mathrm{p}-\mathrm{MTJ}$ s have been extensively characterized. Scaling and switching behavior have been investigated in p-MTJ devices with diameters ranging from $11 \mathrm{~nm}$ to $500 \mathrm{~nm}^{12-16}$, and the effective anisotropy has been estimated by analyzing telegraph noise while measuring TMR using conductive atomic force microscopy ${ }^{17}$.

Applications such as high-density memory require arrays of nanoscale MTJ devices in which good thermal stability, low switching field distribution and low interparticle interactions are desirable characteristics. These properties can be measured by magnetometry of large (mm-scale) area samples, but the limited resolution of photolithography and the limited throughput of electron beam lithography make these patterning methods unsuitable for the fabrication of such samples. Instead, a self-assembly process, block copolymer (BCP) lithography, is convenient for making dense, large-area arrays. Block copolymers can generate well-ordered periodic morphologies on the scale of over $100 \mathrm{~nm}$ to sub-10 $\mathrm{nm}^{18-21}$, and transfer of the self-assembled patterns into magnetic films has been used to form arrays of magnetic wires and dots from a range of materials ${ }^{22-28}$. However, the ensemble behavior of p-MTJ nanoscale arrays has not been explored.

In this article, a CoFeB/MgO/CoFeB p-MTJ was patterned into nanopillar arrays that cover a 0.5 $\mathrm{cm} \times 0.5 \mathrm{~cm}$ sample area using BCP lithography. Both direct and image reversal processes were developed to transfer the structure from the BCP to the p-MTJ stack forming $\mathrm{p}-\mathrm{MTJ}$ nanopillar arrays with pillar diameters of $25 \mathrm{~nm}$ and $64 \mathrm{~nm}$. Magnetic hysteresis measurements were performed as a function of field sweep rate to determine the switching volume and thermal stability, and first-order reversal curves ${ }^{29}$ (FORCs) were used to identify the switching behavior, as well as the magnetostatic interaction between the soft and hard magnetic layers in the $p$ - 
MTJ nanopillars. FORC measurements have been established as a method to characterize the irreversible switching processes as well as the corresponding coercive and interaction field in a range of magnetic systems $s^{30,31}$ that include multilayer thin films $s^{32-34}$ and nanostructure arrays ${ }^{35-}$ 38 .

\section{Experimental methods}

$0.5 \mathrm{~cm} \times 0.5 \mathrm{~cm} \mathrm{MTJ}$ stacks with the structure $\mathrm{Si} / \mathrm{SiO}_{2} / \mathrm{Ta}(6) / \mathrm{Ru}(10) / \mathrm{Ta}(10) /$ $\mathrm{CO}_{20} \mathrm{Fe}_{60} \mathrm{~B}_{20}(0.8) / \mathrm{MgO}(2) / \mathrm{CO}_{20} \mathrm{Fe}_{60} \mathrm{~B}_{20}(1.6) / \mathrm{Ta}(7) / \mathrm{Ru}(8)$ (thicknesses in $\mathrm{nm}$ ), as shown schematically in Figure 1(a), were deposited at ambient temperature by magnetron sputtering with a base pressure of $3 \times 10^{-9}$ Torr. The as-grown MTJ film then underwent a rapid thermal annealing in Ar environment at $300^{\circ} \mathrm{C}$ for 10 minutes to enhance the PMA and TMR. The CoFeB is still substantially amorphous after this annealing process. We found the annealing step must be done before patterning since post-annealing of patterned pillars led to degradation of the magnetic properties, presumably due to oxidation or diffusion at the exposed edges of the pillars.

Two processes, the reverse contrast and direct transfer method that are illustrated in Figure 1(b) and (c) respectively, were used to pattern the annealed p-MTJ film into nanopillar arrays. In the reverse contrast method, a $60 \mathrm{~nm}$ thick polymethyl methacrylate (PMMA) resist layer (950 PMMA, MicroChem, MW = $950 \mathrm{~kg} \mathrm{~mol}^{-1}, 1 \%$ in anisole) was firstly spin-coated onto the $\mathrm{p}$ MTJ film and baked at $130{ }^{\circ} \mathrm{C}$ for 60 seconds, followed by spin-coating a $37 \mathrm{~nm}$ thick polystyrene- $b$-polydimethylsiloxane (PS- $b$-PDMS) BCP (SD75) with molecular weight of $75.5 \mathrm{~kg}$ $\mathrm{mol}^{-1}$ and volume fraction $\sim 41 \%$ PDMS, from a 1 wt\% solution in cyclohexane. The SD75 is synthesized by anionic polymerization. ${ }^{39,40}$ The sample then underwent a 1 hour solvent vapor annealing ${ }^{41}$ in a glass chamber of $5 \mathrm{~cm}$ diameter and $4.5 \mathrm{~cm}$ height, containing $4 \mathrm{~cm}^{3}$ annealing solvent of toluene and heptane made from a 3:1 volumetric liquid mixture. This led to the BCP forming a perforated lamellar morphology (inset image of Figure 2(a)) consisting of the PDMS within a PS matrix, with the PDMS wetting layer at the air surface. After the annealing, $5 \mathrm{~s}$ of $\mathrm{CF}_{4}$ and $50 \mathrm{~s}$ of $\mathrm{O}_{2}$ reactive ion etch were applied to the sample. The $\mathrm{CF}_{4}$ oxidized the PDMS 
surface layer then the $\mathrm{O}_{2}$ removed the PS, oxidized the PDMS perforated lamella to form a silica-like material, and removed the PMMA areas that were not protected by the oxidized PDMS (ox-PDMS). The final structure consisted of a bilayer of ox-PDMS/PMMA containing close-packed pores with center to center spacing of $90 \mathrm{~nm}$. A Pt hard mask was then deposited onto the sample via electron-beam evaporation (base pressure $=7 \times 10^{-7}$ Torr, deposition rate of 5 $\AA \mathrm{s}^{-1}$ ), and liftoff by immersion in n-methyl pyridine (NMP) at $80{ }^{\circ} \mathrm{C}$ for $1 \mathrm{~h}$ removed the PMMA/ox-PDMS/Pt, leaving an array of Pt dots on the MTJ film. Thus the PMMA is a sacrificial layer which enables the ox-PDMS to be removed by NMP, and also provides pores with nearly vertical sidewalls so that the Pt dot features formed at the base of the pores are well defined. The remaining Pt dots were used as a hard mask and the pattern was transferred into the $p$ MTJ stack by Ar ion-beam etching (milling) at 250 volts and $10 \mathrm{~mA}$ beam current. An endpoint detector was used during the etching process to keep track of the sputtered elements, and the etch was stopped immediately after the CoFeB/MgO/CoFeB layers had been etched. This process gives a contrast reversal from perforated lamellae of the BCP to nanopillar arrays of $p$ MTJ.

A direct transfer method, shown in Figure 1(c), was also developed to transfer the pattern from a sphere-forming BCP into the p-MTJ film. First, a $20 \mathrm{~nm}$ thick carbon layer was sputtered onto the p-MTJ film at ambient temperature and then a $35 \mathrm{~nm}$ thick PS- $b$-PDMS layer was spincoated on top of it, with molecular weight of $56 \mathrm{~kg} \mathrm{~mol}^{-1}$ and PDMS volume fraction of $16 \%$ (SD56) yielding a bulk morphology consisting of spheres of PDMS in a PS matrix. After solvent vapor annealing (15 minutes in a toluene vapor environment in the same chamber used for the reverse contrast method), the same reactive ion etch process was used to produce an array of pillars consisting of carbon with ox-PDMS caps. Ar ion beam etching then produced an array of p-MTJ dots capped with C/ox-PDMS.

The patterned arrays were imaged by scanning electron microscopy (Zeiss Sigma) and the geometry was analyzed using the NIH public domain software ImageJ ${ }^{42}$ to calculate dot areas and densities. The magnetic hysteresis loops were measured by alternating gradient 
magnetometry (Princeton MicroMag 2900) with the field sweep rate ranging from $4 \mathrm{Oe} \mathrm{s}^{-1}$ to $800 \mathrm{Oe} \mathrm{s}^{-1}$. Magnetic force microscopy images were taken by a Dimension 3100 Nanoscope IV atomic force microscope with a low moment $\mathrm{CoCr}$ magnetic probe.

\section{Results and Discussion}

Figure 2(a) shows the SEM image after patterning the p-MTJ film from SD75 using the reverse contrast method. The resulting nanopillar arrays have a hexagonal close-packed structure with a periodicity of $90 \pm 2 \mathrm{~nm}$. Assuming the nanopillars to be circular, the diameter distribution calculated from the areas of the dots is plotted in Figure 2(b). The average diameter was $64 \mathrm{~nm}$ and the standard deviation was $4 \mathrm{~nm}$, which is $6 \%$ of the diameter, showing good uniformity of the pattern. The result of the direct transfer method from SD56 is shown in Figures 2(c) and (d). The periodicity is $38 \pm 1 \mathrm{~nm}$ and diameter is $25 \pm 2 \mathrm{~nm}$, i.e. the standard deviation was $8 \%$ of the diameter.

Figure 3 shows the major magnetic hysteresis loops of $5 \mathrm{~mm}$ square samples of the p-MTJ film measured before and after patterning, at a field sweep rate of $25 \mathrm{Oe} \mathrm{s}^{-1}$. The unpatterned film, Fig. 3(a), exhibited PMA with a high remanence, square loop for an out-of-plane field. The upper CoFeB magnetic layer has higher moment and is expected to have a lower coercivity. The out of plane hysteresis loop shows a slope change which may indicate the separate switching of the two layers. The coercivity was $\sim 40$ Oe and the saturation magnetization $\left(M_{s}\right)$ was $492 \mathrm{emu}$ $\mathrm{cm}^{-3}$.

The patterned MTJ nanopillars, Figure $\mathbf{3}(\mathbf{b}-\mathbf{d})$, showed a lower moment per unit area of the substrate, a broader switching field distribution and higher coercivity for an out of plane field than the unpatterned film. The $64 \mathrm{~nm}$ diameter pillars (Figure 3(b) data shown in dark green) exhibited a two-step switching behavior, in which the softer layer switched at 150 Oe and the hard layer switched at $500 \mathrm{Oe}$, with each layer corresponding to about half of the total moment. The standard deviation of switching field distribution ( $\left.\sigma_{S F D}\right)$ for the soft layer was 53 Oe and for the hard layer was 166 Oe. In contrast, for the $25 \mathrm{~nm}$ diameter pillars, no clear step was 
observed in the loop suggesting that the switching field of the two magnetic layers was similar. The coercivity for this sample was 327 Oe and $\sigma_{\text {SFD }}$ was 199 Oe.

The saturation magnetization of the $25 \mathrm{~nm}$ pillars was about $35 \%$ of the value measured in the unpatterned film, which matches the areal coverage of the nanopillars of $37 \%$ based on the SEM images. However, for the $64 \mathrm{~nm}$ pillars, the saturation magnetization was actually lower than the areal coverage of $41 \%$. This situation is caused by the absence of nanopillars in some of the sample area, where non-perforated lamellae or a double-layer morphology was formed by the BCP after solvent vapor annealing and thus there was no Pt hard mask left after liftoff.

First order reversal curves were measured to further characterize the switching behavior of the patterned p-MTJs. For each curve, the measurement began with saturating the sample at $2 \mathrm{kOe}$, then the field was reduced to a reverse field $H_{r}$, and then the ascending loop was measured up to positive saturation. A series of curves was obtained with different $\mathrm{H}_{\mathrm{r}}$. The step sizes of the field $\mathrm{H}$ and $\mathrm{H}_{\mathrm{r}}$ were both $10 \mathrm{Oe}$.

We first consider which magnetization configurations correspond to various points on the FORCs for the sample with $64 \mathrm{~nm}$ diameter pillars, Figure 4(a), and show these schematically in Figure 4(c). The light pink color represents up magnetization and the dark blue color represents down magnetization for the hard (lower) and soft (upper) layers. Points $1-5$ correspond to the descending branch of the loop. At state 1 , both layers of the p-MTJ nanopillars were magnetized in the up (positive) direction. When applying a reverse field, the soft layers switch over a field range of approximately $\mathrm{Hr}_{r}=0$ to $\mathrm{H}_{\mathrm{r}}=-250 \mathrm{Oe}$, including states 2 and 3 . If $\mathrm{Hr}_{r}$ is more negative than $-250 \mathrm{Oe}$, the hard layer in many of the pillars also switched, as in state 4 . When $\mathrm{H}_{\mathrm{r}}$ reached $-800 \mathrm{Oe}$, both layers had switched and were magnetized down, state 5. Based on these configurations we consider the processes that occur while bringing the applied field from each of the states 2, 3, 4 and 5 back to positive saturation. In the case of states 2 and 3, the ascending loop shows a step at around -120 Oe corresponding to the soft layers switching to the up direction. This suggests that the soft layers have an intrinsically low coercivity below $\sim 15$ 
Oe since the midpoint of the step on the descending branch occurred at -150 Oe. The offset from zero is due to the magnetostatic interaction from the hard layer, which will be considered further below. The ascending loop starting from state 4 showed additional steps, as it comprised two groups of pillars. In the first group $(A)$, both layers were magnetized down and in the second group (B), the soft layer was magnetized down but the hard layer was still magnetized up. In the ascending loop, the soft layer of group B switched to up at around -200 Oe, transitioning the sample from state 4 to state 6 . As the applied field further increased, the soft layer of group A begin to switch to the up direction at around +150 Oe, state 7 , followed at higher fields by the hard layer switching back to the up direction, regenerating state 1 . The different switching fields of the soft layer in groups $A$ and $B$ is a result of the different directions of the magnetostatic field produced by the hard layer. Finally, the ascending loop starting from state 5 follows the opposite process of the descending loop from state 1 to state 5 .

Other than the steps in the hysteresis loop discussed above, we observe that the ascending loops exhibit an additional step at around 0 Oe. The magnitude of the magnetization change at this step is greater for the minor loops in which the remanent state contains pillars of both orientation (i.e. pillars with both layers up and pillars with both layers down). It is possible that this feature may represent reversal of the softer material at the edges of the dots, which has lower anisotropy due to the ion beam etch process.

Figure 4(b) plots the FORC distribution defined as

$$
\rho\left(H, H_{r}\right) \equiv-\frac{1}{2} \frac{\partial^{2} M\left(H, H_{r}\right)}{\partial H \partial H_{r}},
$$

where the axes are the coercive field $H_{c 1}=\left(H-H_{r}\right) / 2$ and the interaction field $H_{u}=(H+$ $\left.H_{r}\right) / 2$. The correspondence between Figures $4(a)$ and $4(b)$ is shown by the colour coding. The purple peak at $\mathrm{H}_{\mathrm{c} 1}=100-200$ Oe and the red peak at $\mathrm{H}_{\mathrm{c} 1}=300-350$ Oe represent the switching of the soft layer for pillars in groups $A$ and $B$. The small red peak at $\mathrm{H}_{\mathrm{c} 1}=50$ Oe and the purple peak at $\mathrm{H}_{\mathrm{c} 1}=350$ Oe correspond to the low field feature mentioned above. The large green area at $\mathrm{H}_{\mathrm{u}} \sim 0$ Oe and $\mathrm{H}_{\mathrm{c} 1}=300-750$ Oe is attributed to the hard layer switching. The two peaks that represent the soft layer switching have a difference in interaction field of 150 Oe, 
meaning that the hard layer is exerting a field of $\sim \pm 75$ Oe on the soft layer. This is generally in agreement with our calculation of the magnetostatic field described in a later paragraph.

Figure 5 shows a series of magnetic force microscopy images of the p-MTJ pillars taken at remanence after applying different reverse fields. In (a)-(c), as the reverse field was less than 250 Oe, there were no obvious differences in contrast between pillars. This is consistent with the assumption that the antiparallel state is not stable at remanence, i.e. any soft layers that were switched by the reverse field switched back at remanence due to the magnetostatic interaction from the hard layer. Figure 5 (d) and (e) show the images after applying -400 Oe and -500 Oe reverse field respectively. We start to see a clear contrast between pillars that are magnetized in the up direction and in the down direction, as in state 6 of Figure 4 (c). As the reverse field increases, a larger fraction of pillars is switched to the down direction and all of them were magnetized down when the reverse field exceeded the saturation field (Figure 5(f)). The uniform contrast within each dot supports the interpretation of single domain remanent states.

In contrast, the FORCs of the $25 \mathrm{~nm}$ nanopillars (Figure 6(a)) only showed one magnetization reversal step. Considering the magnetostatic interaction between the soft layer and hard layer, we believe the two layers switch together. However, a small kink was observed in the FORCs at an applied field of 100 Oe, indicating two-step switching in at least some of the pillars. In the corresponding FORC distribution (Figure $6(\mathbf{b})$ ), the peak at $\mathrm{H}_{\mathrm{c} 1}=\sim 50$ Oe relates to the switching of the soft layer and the peak at $\mathrm{H}_{\mathrm{c} 1}=\sim 210$ Oe relates to the switching of the hard layer of those two-step switching pillars, while the ridge at $\mathrm{H}_{\mathrm{c} 1}=\sim 30$ Oe corresponds to pillars in which the two layers switched together.

The magnetostatic interaction between the hard and soft layers is important in determining the reversal behavior of the p-MTJ nanopillars. Assuming the pillar forms a circular disk, we numerically calculate the field by integrating $H=q_{m} / 4 \pi r^{2}$ for both surfaces of the layer, where $q_{m}$ is the magnetic charge on the surface and $r$ is the distance from the charge to the 
target position. Figure 7(a) shows the out-of-plane component of the magnetostatic field originating from the hard layer at the centerline of the soft layer as a function of radius. For 64 $\mathrm{nm}$ pillars, the field varies from 60 Oe at the center to 135 Oe at the edge. Similarly the field produced by the soft layer at the position of the hard layer is plotted in Figure $\mathbf{7}(\mathbf{b})$, showing a higher magnitude. The magnetostatic field in plane away from the edge of the pillars is of opposite direction and decreases rapidly, with a value $<10$ Oe at the position of the nearest neighbor pillars. Similarly, the vertical component of the stray field in $25 \mathrm{~nm}$ p-MTJ pillars is shown in the red curve. Both the fields coming from the soft layer and the hard layer are larger in magnitude as compared to $64 \mathrm{~nm}$ pillars, leading to strong coupling between the two magnetic layers and accounting for the single-step reversal process. As the soft layer reverses, the interaction field exerted on the hard layer changes sign promoting reversal of the hard layer.

We now consider the thermal stability of the p-MTJ pillars following the approach of Sharrock ${ }^{43}$. Due to thermal instability the switching field of small particles varies with the field sweep rate. From the Arrhenius-Néel formulation, the rate of magnetic reversal,

$$
r=f_{0} \exp (-\Delta E / k T)
$$

is defined by the probability per unit time of crossing the energy barrier $\Delta E$. $f_{0}$ is the attempt frequency for barrier crossing and assumed to be on the order of $10^{9} \mathrm{~s}^{-1} . k$ is Boltzmann's constant and $\mathrm{T}$ is temperature in Kelvin. Assuming the Stoner-Wohlfarth (SW) model, the energy barrier can be written as

$$
\Delta E=K V^{*}\left(1-H / H_{0}\right)^{z}
$$

where $\mathrm{K}$ is the magnetic anisotropy, $\mathrm{V}^{*}$ is the effective switching volume and $\mathrm{H}_{0}$ is the field at which the energy barrier $\Delta E$ is zero ${ }^{43} . \mathrm{z}$ is related to the directions of the easy axis and applied field, and according to an approximation made by Pfeiffer ${ }^{44}$,

$$
z \approx 0.86+1.14 x
$$

Here $x(0.5 \leq x \leq 1)$ in equation 3 is the SW parameter determined by the angle $\psi$ between the preferred axis and applied field, namely, 


$$
x=\left[\cos ^{2 / 3}(\psi)+\sin ^{2 / 3}(\psi)\right]^{-3 / 2} .
$$

In addition, from the rate of magnetic reversal in equation 1, the magnetic moment of a collection of identical, noninteracting particles will decay exponentially in time $t$ from a saturated moment $m_{0}$ under a constant opposing field, expressed as

$$
m(t)=m_{0}[2 \exp (-r t)-1]
$$

The relationship between coercivity $\mathrm{H}_{c}$ and time $\mathrm{t}$ can thus be derived,

$$
H_{c}(t)=H_{0}\left\{1-\left[\left(k T / K V^{*}\right) \ln \left(f_{0} t\right)\right]^{n}\right\}
$$

using equations $1,2,5, n \equiv 1 / z$, and the definition of $H_{c}$ that half of the particles were reversed $(m(t)=0)$. By relating the field sweep rate $\mathrm{R}$ to $t$, the effective time during which the field is applied, via $\mathrm{R}=-d H / d t$, we obtain

$$
t=\frac{1}{R} n H_{0}\left[\ln \left(f_{0} t\right)\right]^{n-1}\left(k T / K V^{*}\right)^{n}
$$

Equations 6 and 7 can be fitted to experimental data $H_{c}(R)$ to obtain $K, V^{*}$ and $H_{0}$.

The hysteresis loops of patterned p-MTJ nanopillar arrays under different field sweep rate were measured by AGM and are plotted in Figures 8(a) and 8(c). For $64 \mathrm{~nm}$ pillars, the coercivity of the soft layer did not show an obvious change with field sweep rate, but that of the hard layer increased from $481 \pm 5$ Oe to $692 \pm 8$ Oe as the field sweep rate increased from 4 Oe $\mathrm{s}^{-1}$ to 800 Oe $\mathrm{s}^{-1}$. It is important to point out here that the field shown in the hysteresis loops is the external applied field from the AGM, but to estimate the thermal stability characteristics, the actual field experienced by the sample population should be considered. This includes the magnetostatic field from adjacent layers and neighboring pillars in addition to the external applied field. Based on this, the actual coercivity of the hard layer changed from 631 Oe at a field sweep rate of 4 $\mathrm{Oe} \mathrm{s}^{-1}$ to 842 Oe at $800 \mathrm{Oe} \mathrm{s}^{-1}$, taking into account the 150 Oe magnetostatic field from the soft layer in addition to the external applied field. We then evaluate the thermal stability from the numerical solution of the effective time $t$ in equation 7 , assuming $n=0.614\left(\psi=10^{\circ}\right)$, and starting from $\mathrm{H}_{0}=5000 \mathrm{Oe}$ and $\mathrm{KV} * / \mathrm{kT}=40$. A value of $\mathrm{n}=0.614$, rather than $\mathrm{n}=0.5$ for $\psi=0$, 
was adopted to account for deviations in the direction of the easy axis of the pillars. By iterating between equations 6 and 7 , more refined values of $\mathrm{KV}^{*}$ and $\mathrm{H}_{0}$ were obtained, and $\mathrm{V}^{*}$ was then obtained using $\mathrm{H}_{0}=2 x \mathrm{~K} / \mathrm{M}_{\mathrm{s}}$. Figure $\mathbf{8}(\mathbf{b})$ shows the fitting of equation 6 and the results are listed in Table 1. The estimated thermal stability factor $\Delta\left(\mathrm{KV}^{*} / \mathrm{kT}\right)$ was $43.5 \pm 1.1$ and the magnetic anisotropy $\mathrm{K}$ was $8.15 \times 10^{5} \mathrm{erg}^{-3}$. We independently obtained $\mathrm{K}$ by measuring the area between the hard axis loop and the magnetization axis, and subtracting the corresponding area from the easy axis loop, as shown in Figure $\mathbf{3}(\mathbf{c}, \mathbf{d})$. This yielded $6.42 \times 10^{5} \mathrm{erg} \mathrm{cm}^{-3}$, which is a reasonable agreement considering the approximations of the model. The deviation may come from the assumption of Stoner-Wohlfarth reversal or from the switching field distribution of the dots which leads to a range of thermal stability factors within the ensemble. Nevertheless, both estimates generally agree with the values reported previously ${ }^{24,28}$.

The value of $\mathrm{K}$ allows an estimate of the domain wall width $\delta_{\mathrm{w}}$ which in the limit of zero film thickness can be estimated by $\delta_{w}=\pi \sqrt{ }(A / K)$, where $\mathrm{A}$ is the exchange stiffness ${ }^{45}$ taken as $\sim 19$ pJ $\mathrm{m}^{-146}$. Using $\mathrm{K}=8.15 \times 10^{5} \mathrm{erg} \mathrm{cm}^{-3}$ yields $\delta_{\mathrm{w}} \sim 48 \mathrm{~nm}$, which implies that domain walls are unlikely to exist in the patterned pillars.

From the thermal stability factor and estimated magnetic anisotropy, the effective switching volume $\mathrm{V}^{*}$ is calculated as $2.19 \times 10^{3} \mathrm{~nm}^{3}$ at room temperature. The smaller $\mathrm{V}^{*}$ compared to the actual volume of the hard layer (radius $a=32 \mathrm{~nm}$, height $\mathrm{h}=1.6 \mathrm{~nm}, \pi \mathrm{a}^{2} \mathrm{~h}=2.57 \times 10^{3} \mathrm{~nm}^{3}$ ) is consistent with the speculation of incoherent reversal, e.g. from edge-nucleated reversal of the pillars.

The soft layer showed little scan rate dependence of its coercivity. The actual coercivity of the soft layer was estimated to be 10-70 Oe after subtracting the magnetostatic field from the hard layer, which opposes the external field as the soft layers switch in the descending loop. The range of accessible sweep rates in the AGM was insufficient to yield a significant sweep rate dependent coercivity. 
For the $25 \mathrm{~nm}$ pillars, since the hysteresis loops showed a single step switching behavior, we estimate the thermal stability by considering a pillar as a single magnetic entity. From table 1, the thermal stability factor was $\mathrm{KV}^{*} / \mathrm{kT}=28.1 \pm 0.2$ and the magnetic anisotropy $\mathrm{K}=4.41 \times 10^{5}$ erg $\mathrm{cm}^{-3}$. In comparison, the $\mathrm{K}$ determined by the area difference between the easy and hard axis hysteresis loops (shown in $\mathrm{SI}$ ) is $9.49 \times 10^{5} \mathrm{erg} \mathrm{cm}^{-3}$. The effective switching volume was estimated as $2.62 \times 10^{3} \mathrm{~nm}^{3}$, which is larger than the volume of one pillar (radius $=12.5 \mathrm{~nm}$, height $\left.=2.4 \mathrm{~nm}, \pi \mathrm{a}^{2} \mathrm{~h}=1.18 \times 10^{3} \mathrm{~nm}^{3}\right)$. This may be a result of magnetostatic interactions between pillars, leading to correlated reversal, or it may reflect the dominance of only part of the population of pillars in the sweep rate dependent measurements.

In conclusion, CoFeB/MgO/CoFeB p-MTJ nanopillars with diameters of $64 \mathrm{~nm}$ and $25 \mathrm{~nm}$ were obtained using block copolymer patterning. Pattern transfer processes were developed to yield dot arrays from a perforated lamella or a sphere array, which include the use of a PMMA sacrificial layer to facilitate liftoff in the reverse contrast process, and a carbon hard mask for ion beam etching in the direct process. Magnetostatic interactions play an important role in coupling the hard and soft layers, especially for the smaller pillars. The $64 \mathrm{~nm}$ diameter $\mathrm{p}$-MTJ pillars exhibit a field plateau within which the two layers are antiparallel, and a thermal stability factor KV/kT of the hard layer exceeding 40, which corresponds to 10 years. However, the 25 $\mathrm{nm}$ diameter pillars have lower thermal stability and the two layers do not switch independently due to the dominant effect of interlayer interaction. This coupling can be reduced by using a synthetic antiferromagnetic hard layer to lower the magnetostatic field on the soft layer, or by increasing the PMA, e.g. by adding a thin Mo layer between Ta and CoFeB ${ }^{47}$. The results are relevant to the scaling of MTJ nanostructures for memory and logic devices.

Support from C-SPIN, a STARnet Center of MARCO and DARPA, is gratefully acknowledged. This work used facilities of CMSE, an NSF MRSEC under award DMR 1419807. 
Figures:

(a)

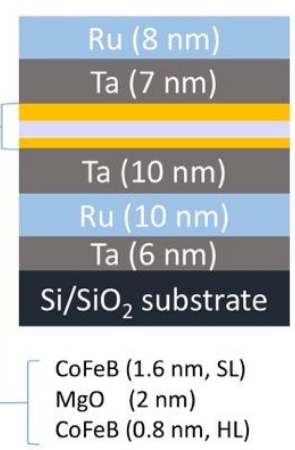

(b)
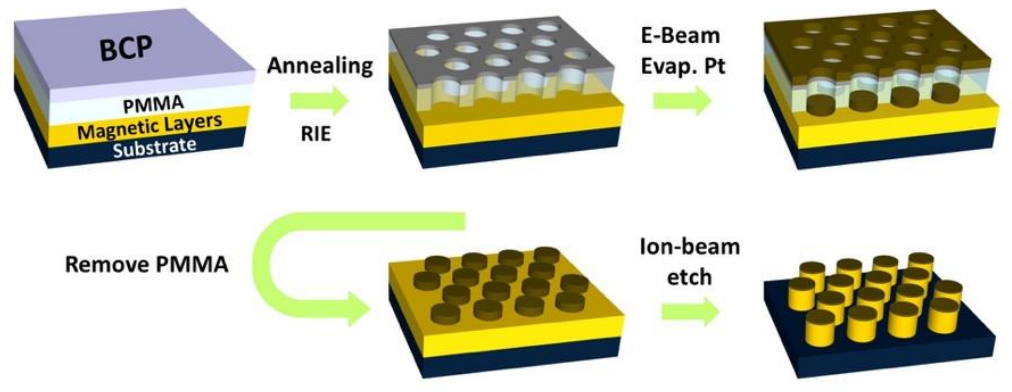

(c)

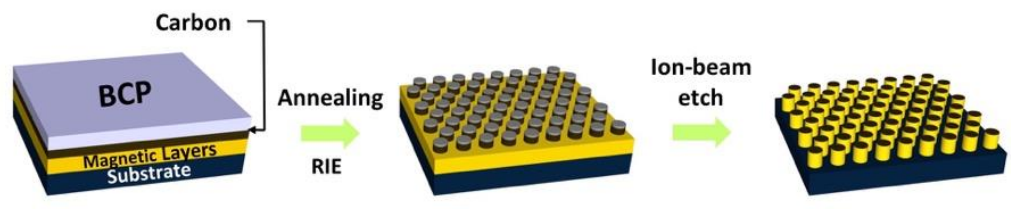

Figure 1. (a) A schematic of the layered structure of the perpendicular magnetic tunnel junction used in this work. SL and HL are the soft and hard layers respectively. (b)(c) Block copolymer patterning process for the magnetic nanostructure. In (b) the perforated lamellar BCP pattern is transferred to the magnetic thin film with inverse contrast, while in (c) the sphere BCP pattern is transferred directly. 
(a)

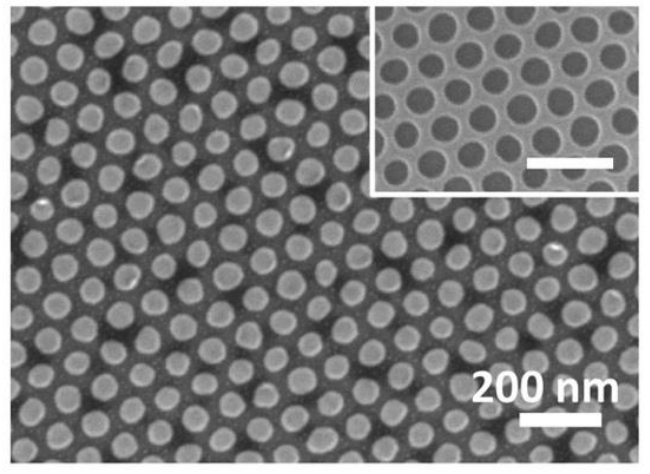

(b)

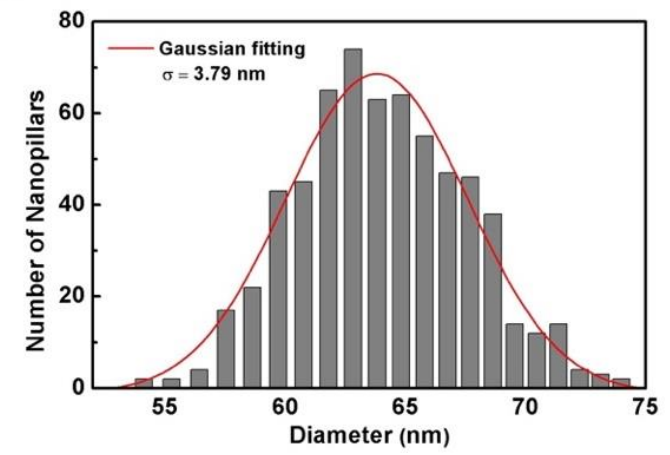

(c)

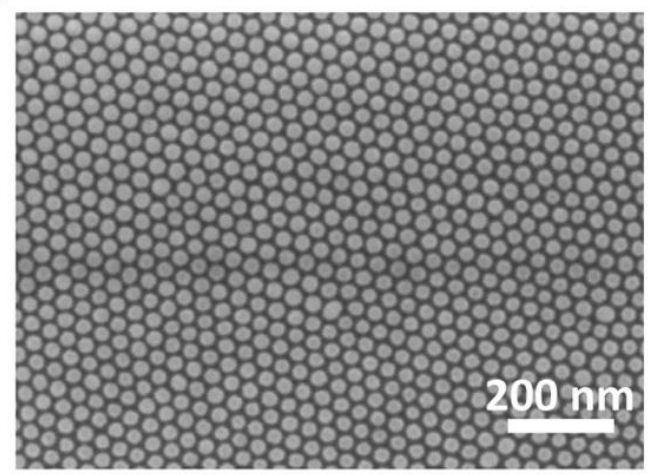

(d)

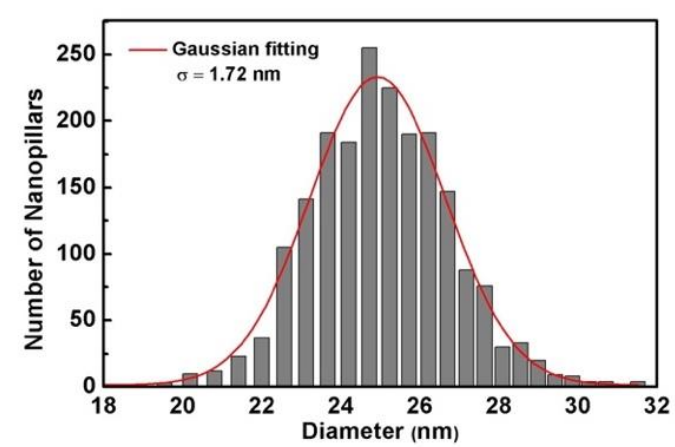

Figure 2. (a) Scanning electron microscope images of $64 \mathrm{~nm}$ diameter p-MTJ nanopillars. The inset (scale bar: $200 \mathrm{~nm}$ ) shows the perforated lamellar BCP pattern of SD75 used for inverse transfer method. (b) The distribution of pillar diameters in (a). (c) Scanning electron microscope images of $25 \mathrm{~nm}$ diameter p-MTJ nanopillars made using SD56 as a mask. (d) The distribution of pillar diameters in (c). 
(a)

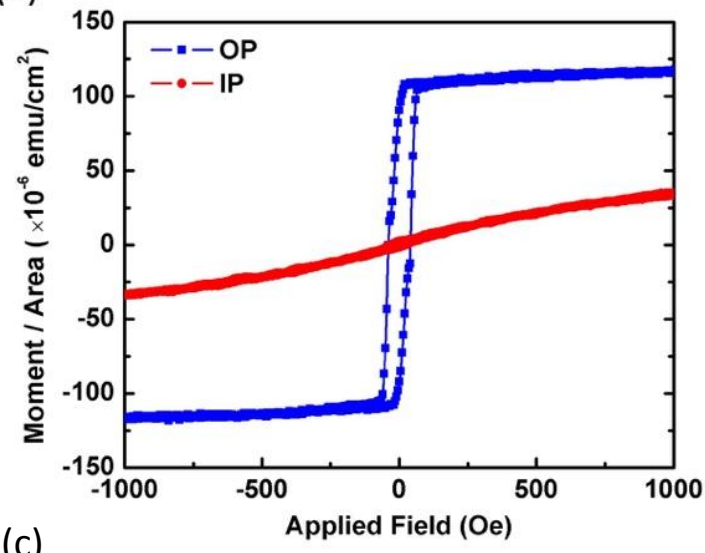

(c)

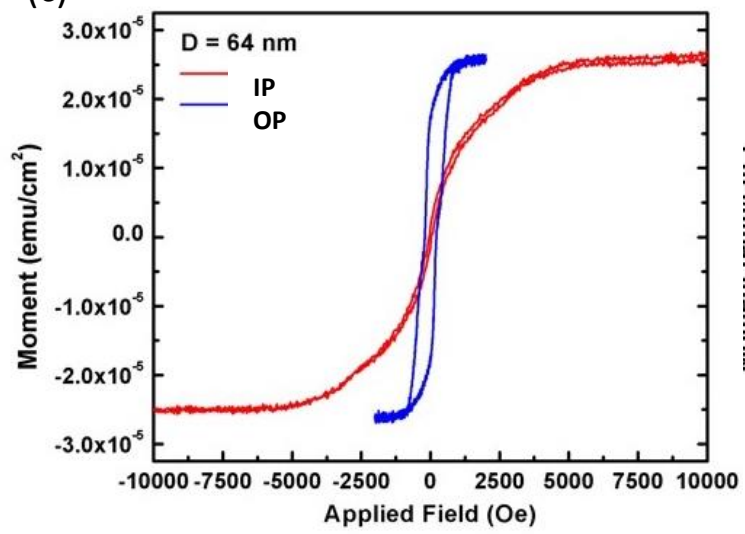

(b)

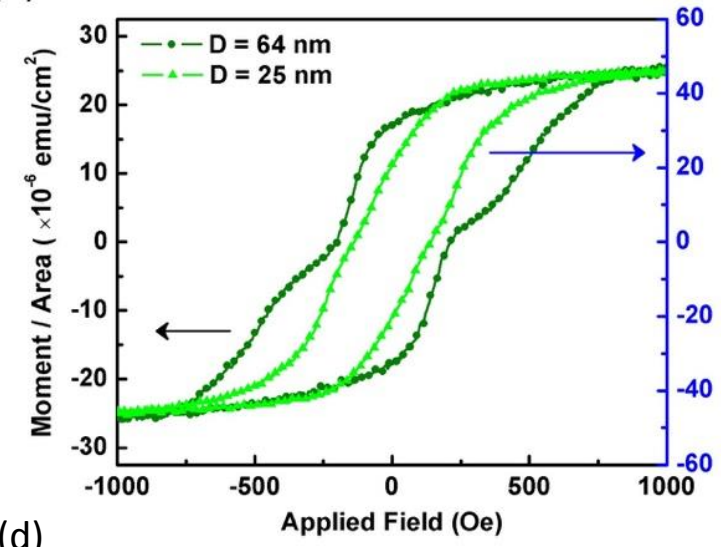

(d)

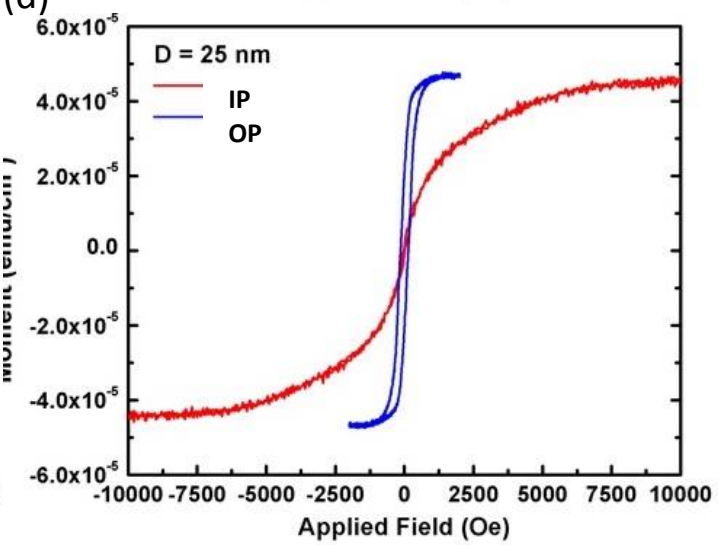

Figure 3. (a) In-plane and out-of-plane hysteresis loop of unpatterned p-MTJ thin film, showing perpendicular magnetic anisotropy. (b) Out-of-plane hysteresis loops of p-MTJ nanopillars with diameter of 64 and $25 \mathrm{~nm}$. Note different scales on the vertical axis. (c,d) In-plane and out-ofplane hysteresis loops of (c) $64 \mathrm{~nm}$ pillar and (d) $25 \mathrm{~nm}$ pillar arrays, with a larger field axis scale. 
(a)

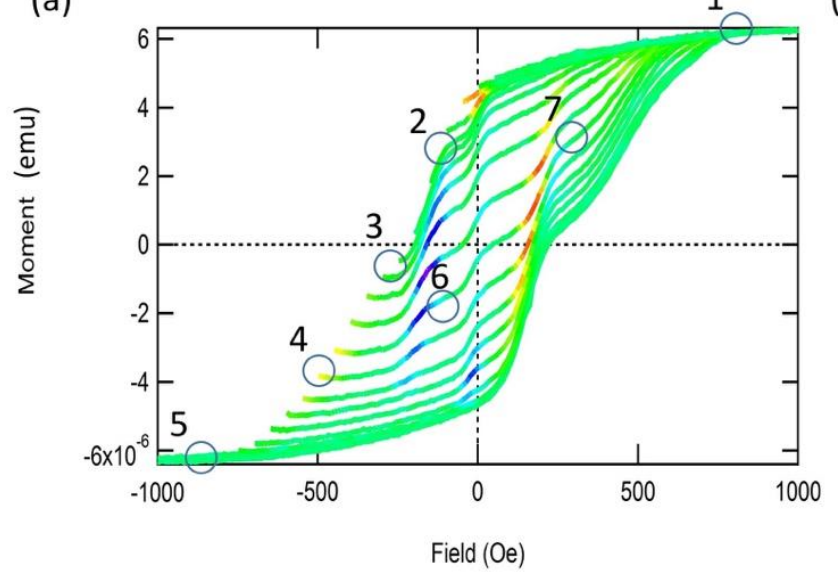

(b)

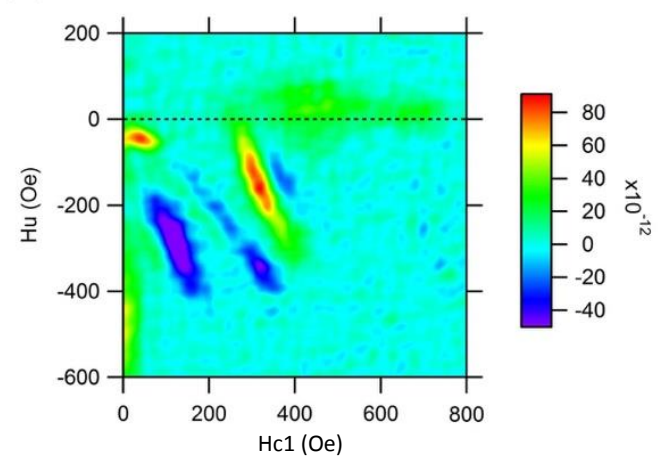

(c)

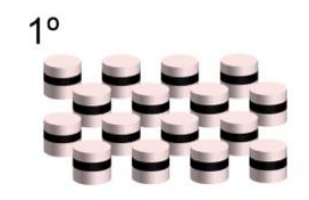

$2^{\circ}$

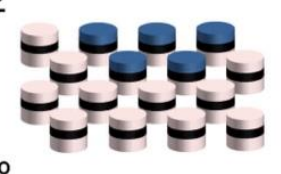

$7^{\circ}$
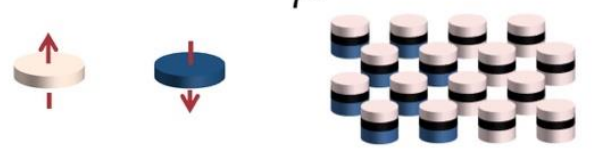

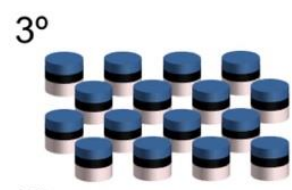

$6^{\circ}$

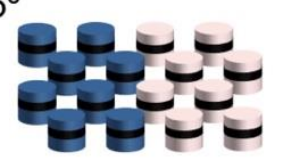

$4^{\circ}$

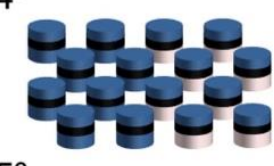

$5^{\circ}$

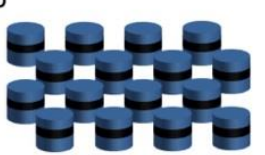

Figure 4. (a) First-order reversal curves of $64 \mathrm{~nm} \mathrm{p-MTJ}$ pillars, showing only $1 / 10$ of all the measured curves for clarity. (b) The corresponding FORC distribution. (c) Schematics of the magnetic configuration of patterned nanopillar arrays. The numbers represent different configurations in (a). The soft layer is the upper layer. 

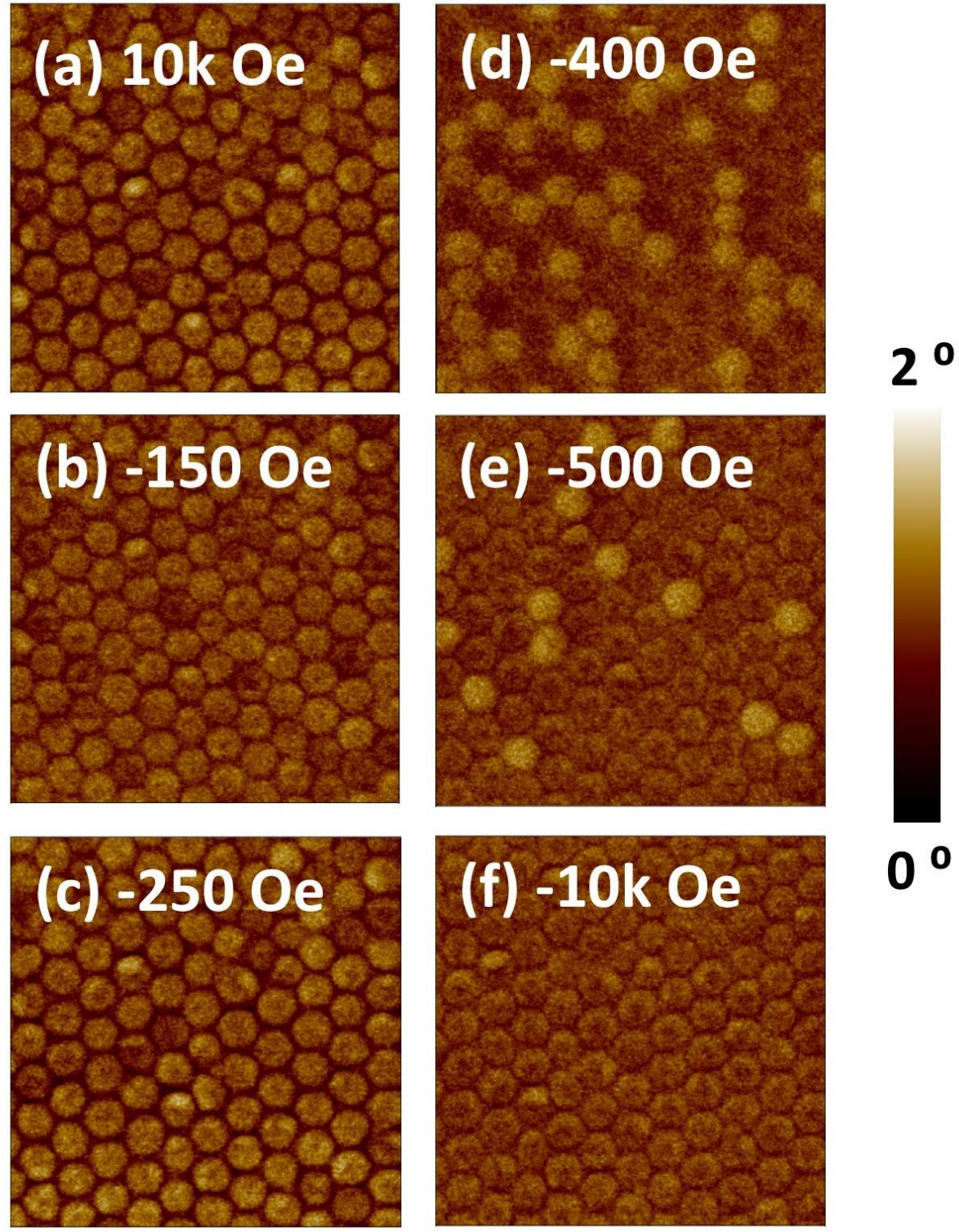

$0^{0}$

Figure 5. Magnetic force microscope images of $64 \mathrm{~nm}$ p-MTJ pillars. The field of view is $1 \mu \mathrm{m} \mathrm{x}$ $1 \mu \mathrm{m}$. (a)-(f) Images taken at remanence after saturating then applying different reverse fields. $(d, e)$ show clear evidence of coexisting up and down net magnetic moment. 
(a)

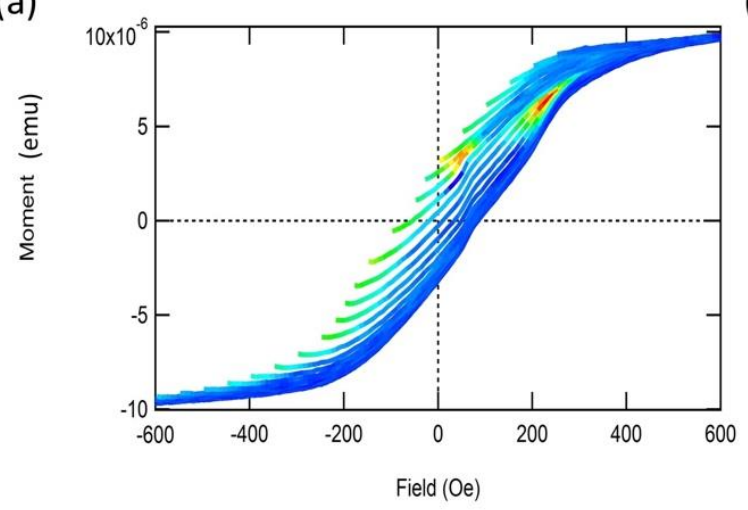

(b)

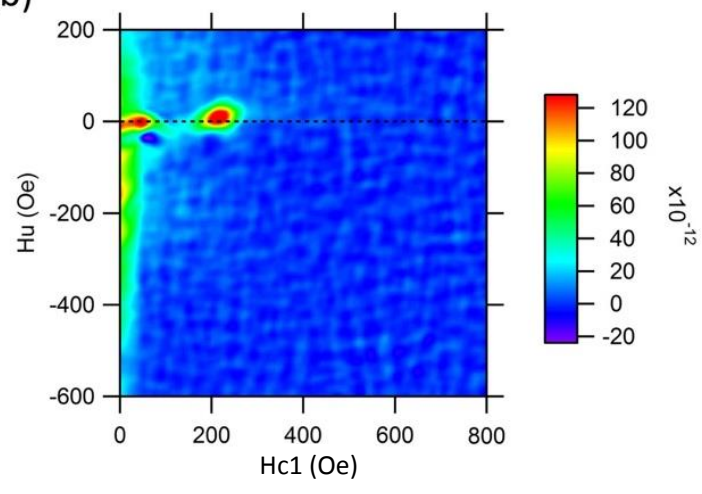

Figure 6. (a) First-order reversal curves of $25 \mathrm{~nm} \mathrm{p-MTJ}$ pillars, showing only 1/10 of the measured curves for clarity. (b) The corresponding FORC distribution of (a).

(a)

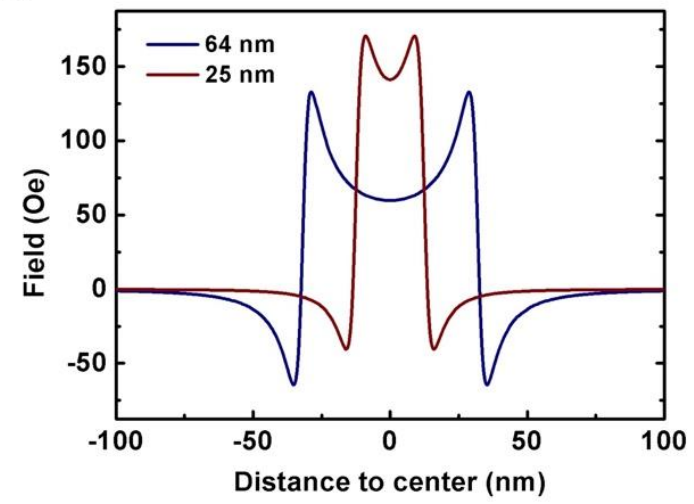

(b)

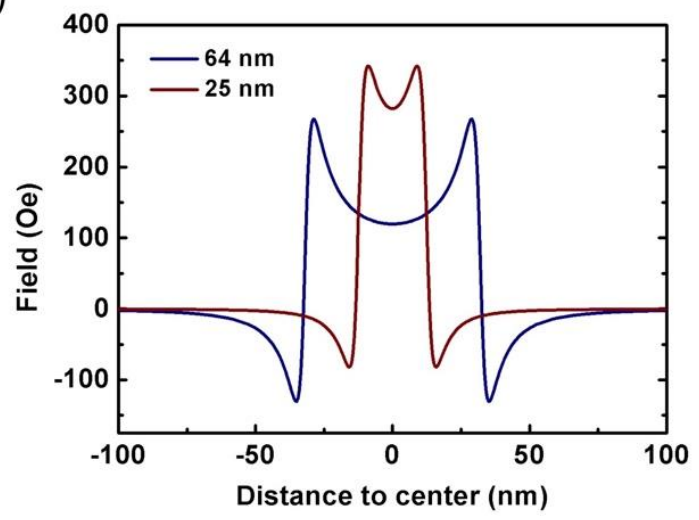

Figure 7. Calculated out-of-plane magnetostatic field distribution of (a) the hard layer at the midline of the soft layer and (b) the soft layer at the midline of the hard layer. 
(a)

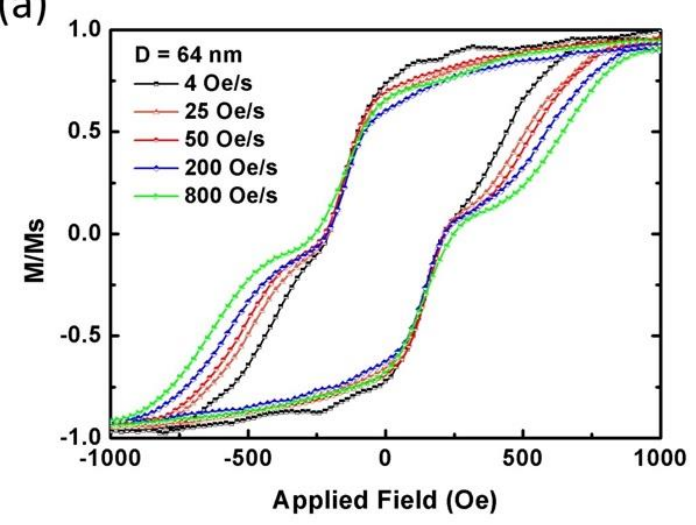

(c)

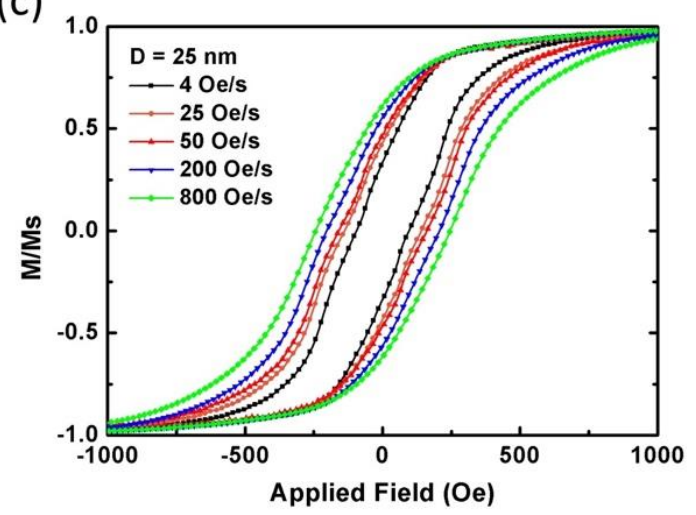

(b)

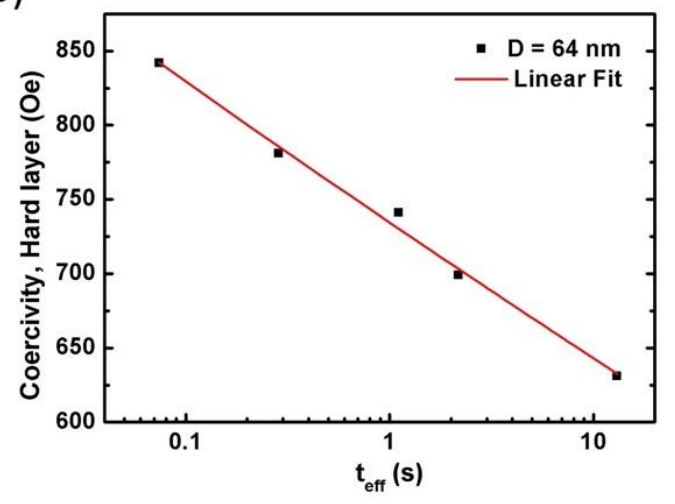

(d)

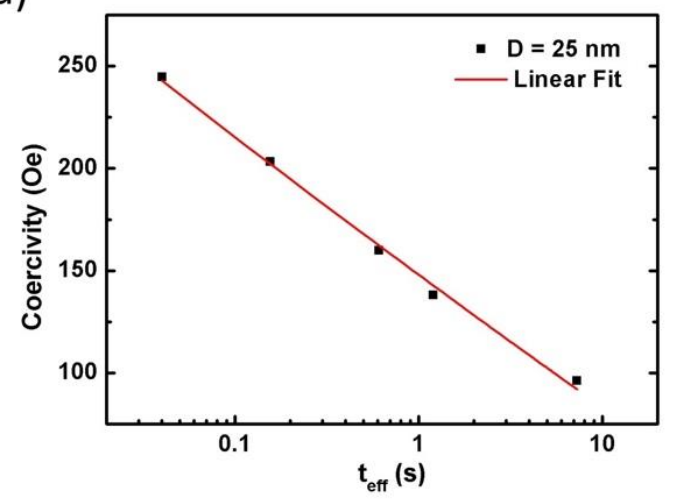

Figure 8. The hysteresis loops of (a) $64 \mathrm{~nm}$ and (c) $25 \mathrm{~nm}$ nanopillar arrays measured under different field sweeping rate. (b)(d) The linear fitting of the variation of coercivity with respect to log of the scan time $t$ (inversely proportional to the field sweep rate), from which the thermal stability factor and $\mathrm{H}_{0}$ are obtained using eq. 8 . 
Table 1. Estimation of the magnetic properties of BCP patterned p-MTJ nanopillars.

\begin{tabular}{|c|c|c|c|c|c|c|}
\hline & $\begin{array}{c}K \\
\left(\mathrm{erg} / \mathrm{cm}^{3}\right)\end{array}$ & $\begin{array}{c}\text { K (from } \\
\text { hys. loop) } \\
\left(\mathrm{erg} / \mathrm{cm}^{3}\right)\end{array}$ & $\begin{array}{c}V^{*} \\
\left(n m^{3}\right)\end{array}$ & $\begin{array}{c}\text { Pillar V } \\
\left(n m^{3}\right)\end{array}$ & $\begin{array}{c}H_{k} \text { (fitted) } \\
\quad(O e)\end{array}$ & $\begin{array}{l}\text { Thermal } \\
\text { stability } \\
\text { factor } \Delta\end{array}$ \\
\hline $\begin{array}{c}64 \mathrm{~nm} \\
\text { Hard Layer }\end{array}$ & $8.15 \times 10^{5}$ & $6.42 \times 10^{5}$ & $2.195 \times 10^{3}$ & $2.574 \times 10^{3}$ & $3305 \pm 99$ & $43.5 \pm 1.1$ \\
\hline $25 \mathrm{~nm}$ & $4.41 \times 10^{5}$ & $9.49 \times 10^{5}$ & $2.620 \times 10^{3}$ & $1.178 \times 10^{3}$ & $1789 \pm 55$ & $28.1 \pm 0.2$ \\
\hline
\end{tabular}




\section{References}

1. Ikeda, S. et al. A perpendicular-anisotropy CoFeB-MgO magnetic tunnel junction. Nat. Mater. 9, 721-724 (2010).

2. Nowak, J. J. et al. Demonstration of Ultralow Bit Error Rates for Spin-Torque Magnetic Random-Access Memory With Perpendicular Magnetic Anisotropy. IEEE Magn. Lett. 2, 3000204-3000204 (2011).

3. Thomas, L. et al. Perpendicular spin transfer torque magnetic random access memories with high spin torque efficiency and thermal stability for embedded applications (invited). J. Appl. Phys. 115, 172615 (2014).

4. Wang, W. G. et al. In-situ characterization of rapid crystallization of amorphous CoFeB electrodes in $\mathrm{CoFeB} / \mathrm{MgO} / \mathrm{CoFeB}$ junctions during thermal annealing. Appl. Phys. Lett. 95, 242501 (2009).

5. Wang, W. et al. Rapid thermal annealing study of magnetoresistance and perpendicular anisotropy in magnetic tunnel junctions based on $\mathrm{MgO}$ and CoFeB. Appl. Phys. Lett. 99, 102502 (2011).

6. Almasi, H. et al. Enhanced tunneling magnetoresistance and perpendicular magnetic anisotropy in Mo/CoFeB/MgO magnetic tunnel junctions. Appl. Phys. Lett. 106, 182406 (2015).

7. Almasi, H. et al. Perpendicular magnetic tunnel junction with $\mathrm{W}$ seed and capping layers. J. Appl. Phys. 121, 153902 (2017).

8. Peng, S. et al. Origin of interfacial perpendicular magnetic anisotropy in $\mathrm{MgO} / \mathrm{CoFe} /$ metallic capping layer structures. Sci. Rep. 5, 18173 (2016).

9. Wang, W.-G., Li, M., Hageman, S. \& Chien, C. L. Electric-field-assisted switching in magnetic tunnel junctions. Nat. Mater. 11, 64-68 (2012).

10. Wang, W. G. \& Chien, C. L. Voltage-induced switching in magnetic tunnel junctions with perpendicular magnetic anisotropy. J. Phys. D. Appl. Phys. 46, 74004 (2013).

11. Newhouse-Illige, T. et al. Voltage-controlled interlayer coupling in perpendicularly magnetized magnetic tunnel junctions. Nat. Commun. 8, 15232 (2017).

12. Sato, H. et al. Properties of magnetic tunnel junctions with a MgO / CoFeB / Ta / CoFeB / $\mathrm{MgO}$ recording structure down to junction diameter of $11 \mathrm{~nm}$. Appl. Phys. Lett. 62403, 2012-2016 (2014).

13. Chenchen, J. W. et al. Size Dependence Effect in MgO-Based CoFeB Tunnel Junctions with Perpendicular Magnetic Anisotropy. Jpn. J. Appl. Phys. 51, 13101 (2012).

14. Tryputen, L. et al. Patterning of sub-50 nm perpendicular CoFeB/MgO-based magnetic tunnel junctions. Nanotechnology 27, 185302 (2016).

15. Wang, W. G. et al. Parallel fabrication of magnetic tunnel junction nanopillars by nanosphere lithography. Sci. Rep. 3, 1948 (2013).

16. Piotrowski, S. K. et al. Conductive Atomic Force Microscopy of Small Magnetic Tunnel Junctions With Interface Anisotropy. IEEE Trans. Magn. 51, 4400504 p1-4 (2015).

17. Bapna, M. et al. Magnetostatic effects on switching in small magnetic tunnel junctions. Appl. Phys. Lett. 108, 022406 p1-4 (2016).

18. Lane, A. P. et al. Directed Self-Assembly and Pattern Transfer of Five Nanometer Block Copolymer Lamellae. ACS Nano 11, 7656-7665 (2017). 
19. Kennemur, J. G., Yao, L., Bates, F. S. \& Hillmyer, M. a. Sub-5 nm Domains in Ordered Poly(cyclohexylethylene)- block -poly(methyl methacrylate) Block Polymers for Lithography. Macromolecules 47, 1411-1418 (2014).

20. Bai, W. et al. Thin Film Morphologies of Bulk-Gyroid Polystyrene- block polydimethylsiloxane under Solvent Vapor Annealing. Macromolecules 47, 6000-6008 (2014).

21. Stoykovich, M. P. \& Nealey, P. F. Block copolymers and conventional lithography. Mater. Today 9, 20-29 (2006).

22. Ross, C. A. \& Cheng, J. Y. Patterned Magnetic Media Made by Self-Assembled BlockCopolymer Lithography. MRS Bull. 33, 838-845 (2008).

23. Kamata, Y., Kikitsu, A., Hieda, H., Sakurai, M. \& Naito, K. Ar ion milling process for fabricating CoCrPt patterned media using a self-assembled PS-PMMA diblock copolymer mask. J. Appl. Phys. 95, 6705-6707 (2004).

24. Hellwig, O. et al. Bit patterned media based on block copolymer directed assembly with narrow magnetic switching field distribution. Appl. Phys. Lett. 96, 52511 (2010).

25. Terris, B. D. \& Thomson, T. Nanofabricated and self-assembled magnetic structures as data storage media. J. Phys. D. Appl. Phys. 38, R199-R222 (2005).

26. Cheng, J. Y. et al. Formation of a Cobalt Magnetic Dot Array via Block Copolymer Lithography. Adv. Mater. 13, 1174-1178 (2001).

27. Jung, Y. S., Lee, J. H., Lee, J. Y. \& Ross, C. A. Fabrication of diverse metallic nanowire arrays based on block copolymer self-assembly. Nano Lett. 10, 3722-3726 (2010).

28. Ho, P. et al. Domain configurations in Co/Pd and L1 0 -FePt nanowire arrays with perpendicular magnetic anisotropy. Nanoscale 8, 5358-5367 (2016).

29. Pike, C. R. First-order reversal-curve diagrams and reversible magnetization. Phys. Rev. $B$ 68, 104424 (2003).

30. Roberts, A. P., Pike, C. R. \& Verosub, K. L. First-order reversal curve diagrams: A new tool for characterizing the magnetic properties of natural samples. J. Geophys. Res. Solid Earth 105, 28461-28475 (2000).

31. Winklhofer, M., Dumas, R. K. \& Liu, K. Identifying reversible and irreversible magnetization changes in prototype patterned media using first- and second-order reversal curves. J. Appl. Phys. 103, 07 C508 (2008).

32. Davies, J. E. et al. Magnetization reversal of Co/Pt multilayers: Microscopic origin of highfield magnetic irreversibility. Phys. Rev. B - Condens. Matter Mater. Phys. 70, 224434 (2004).

33. Davies, J. E. et al. Reversal mode instability and magnetoresistance in perpendicular (Co/Pd)/Cu/(Co/Ni) pseudo-spin-valves. Appl. Phys. Lett. 103, 22409 (2013).

34. Davies, J. E., Hellwig, O., Fullerton, E. E. \& Liu, K. Temperature-dependent magnetization reversal in (Co Pt) Ru multilayers. Phys. Rev. B - Condens. Matter Mater. Phys. 77, 1-7 (2008).

35. Pike, C. R., Ross, C. A., Scalettar, R. T. \& Zimanyi, G. First-order reversal curve diagram analysis of a perpendicular nickel nanopillar array. Phys. Rev. B 71, 134407 (2005).

36. Tiberto, P. et al. Magnetization reversal and microstructure in polycrystalline Fe $50 \mathrm{Pd} 50$ dot arrays by self-assembling of polystyrene nanospheres. Sci. Technol. Adv. Mater. 17, 462-472 (2016). 
37. Rahman, M. T. et al. Controlling magnetization reversal in Co/Pt nanostructures with perpendicular anisotropy. Appl. Phys. Lett. 94, 42507 (2009).

38. Ognev, A. V et al. Self-organization and FORC-based magnetic characterization of ultrahigh aspect ratio epitaxial Co nanostrips produced by oblique deposition on an ordered step-bunched silicon surface. Nanotechnology 28, 95708 (2017).

39 N. Politakos, E. Ntoukas, A. Avgeropoulos, V. Krikorian, B. D. Pate, E. L. Thomas, R. M. Hill, Strongly segregated cubic microdomain morphology consistent with the double gyroid phase in high molecular weight diblock copolymers of polystyrene and poly(dimethylsiloxane), J. Polym. Sci. Part B: Polym. Phys. 47, 2419 (2009)

40. B. M.D. O’Driscoll, R. A. Kelly, M. Shaw, P. Mokarian-Tabari, G. Liontos, K. Ntetsikas, A. Avgeropoulos, N. Petkov, M. A. Morris, Achieving structural control with thin polystyrene-b-polydimethylsiloxane block copolymer films: The complex relationship of interface chemistry, annealing methodology and process conditions, Eur. Polym. J. 49, 3445 (2013)

41. Tu, K., Bai, W., Liontos, G. \& Ntetsikas, K. Universal pattern transfer methods for metal nanostructures by block copolymer lithography. Nanotechnology 26, 375301 (2015)

42. Schneider, Caroline A., Wayne S. Rasband, and Kevin W. Eliceiri. "NIH Image to ImageJ: 25 years of image analysis." Nature methods 9 671-675 (2012)

43. Sharrock, M. P. Measurement and interpretation of magnetic time effects in recording media. IEEE Trans. Magn. 35, 4414-4422 (1999).

44. Pfeiffer, H. Determination of anisotropy field distribution in particle assemblies taking into account thermal fluctuations. Phys. status solidi 118, 295-306 (1990).

45. Schlömann, E. Domain walls in bubble films. I. General theory of static properties. J. Appl. Phys. 44, 1837-1849 (1973).

46. Sato, H., Yamanouchi, M. \& Miura, K. CoFeB thickness dependence of thermal stability factor in $\mathrm{CoFeB} / \mathrm{MgO}$ perpendicular magnetic tunnel junctions. IEEE Magn. Lett. 3, 3000204 p1-4 (2012).

47. Almasi, H., Xu, M., Xu, Y., Newhouse-Illige, T. \& Wang, W. G. Effect of Mo insertion layers on the magnetoresistance and perpendicular magnetic anisotropy in $\mathrm{Ta} / \mathrm{CoFeB} / \mathrm{MgO}$ junctions. Appl. Phys. Lett. 109, 32401 (2016). 